\title{
Single-particle motion in colloids: force-induced diffusion
}

\author{
ROSEANNA N. ZIA ${ }^{1} \dagger$ AND JOHN F. BRADY $Y^{1,2}$ \\ ${ }^{1}$ Department of Mechanical Engineering, California Institute of Technology, Pasadena, CA 91125, USA \\ ${ }^{2}$ Department of Chemical Engineering, California Institute of Technology, Pasadena, CA 91125, USA
}

(Received 7 July 2009; revised 26 March 2010; accepted 29 March 2010;

first published online 9 June 2010)

We study the fluctuating motion of a Brownian-sized probe particle as it is dragged by a constant external force through a colloidal dispersion. In this nonlinearmicrorheology problem, collisions between the probe and the background bath particles, in addition to thermal fluctuations of the solvent, drive a long-time diffusive spread of the probe's trajectory. The influence of the former is determined by the spatial configuration of the bath particles and the force with which the probe perturbs it. With no external forcing the probe and bath particles form an equilibrium microstructure that fluctuates thermally with the solvent. Probe motion through the dispersion distorts the microstructure; the character of this deformation, and hence its influence on the probe's motion, depends on the strength with which the probe is forced, $F^{e x t}$, compared to thermal forces, $k T / b$, defining a Péclet number, $P e=F^{e x t} /(k T / b)$, where $k T$ is the thermal energy and $b$ the bath particle size. It is shown that the long-time mean-square fluctuational motion of the probe is diffusive and the effective diffusivity of the forced probe is determined for the full range of Péclet number. At small $\mathrm{Pe}$ Brownian motion dominates and the diffusive behaviour of the probe characteristic of passive microrheology is recovered, but with an incremental flow-induced 'microdiffusivity' that scales as $D^{\text {micro }} \sim D_{a} P e^{2} \phi_{b}$, where $\phi_{b}$ is the volume fraction of bath particles and $D_{a}$ is the self-diffusivity of an isolated probe. At the other extreme of high Péclet number the fluctuational motion is still diffusive, and the diffusivity becomes primarily force induced, scaling as $\left(F^{e x t} / \eta\right) \phi_{b}$, where $\eta$ is the viscosity of the solvent. The force-induced microdiffusivity is anisotropic, with diffusion longitudinal to the direction of forcing larger in both limits compared to transverse diffusion, but more strongly so in the high-Pe limit. The diffusivity is computed for all $P e$ for a probe of size $a$ in a bath of colloidal particles, all of size $b$, for arbitrary size ratio $a / b$, neglecting hydrodynamic interactions. The results are compared with the force-induced diffusion measured by Brownian dynamics simulation. The theory is also compared to the analogous shear-induced diffusion of macrorheology, as well as to experimental results for macroscopic falling-ball rheometry. The results of this analysis may also be applied to the diffusive motion of self-propelled particles.

Key words: colloids, particle/fluid flow, rheology, Stokesian dynamics, suspensions

\section{Introduction}

The motion of active microscale particles driven through complex fluids is a physical process central to many current scientific problems: vesicle trafficking in cells, artificial

$\dagger$ Email address for correspondence: roseanna@caltech.edu 
nano-motors and nanotherapeutic drug delivery devices are a few important examples (Janke et al. 2005; Shirai et al. 2005; Heath, Davis \& Hood 2009). The increased demand for knowledge of this small-scale behaviour has made microrheology a key step in the understanding, use and design of such systems. Among the collection of techniques known as microrheology, most involve tracking the movement of a colloidal particle (or a set of particles) in order to determine the properties of the surrounding environment (MacKintosh \& Schmidt 1999). There are two main types of particle tracking microrheology: passive - tracking the random motion due to thermal fluctuations - and active - applying a constant or oscillatory force to the particles, for example by using optical tweezers or magnetic fields. A detailed comparison can be found in Khair \& Brady (2006). Most microrheological work to date has focused on passive microrheology, to obtain linear viscoelastic properties by correlating the random thermally driven displacements of tracers to the complex modulus through a generalized Stokes-Einstein-Sutherland relation - a process which is well understood but limited in its scope to equilibrium systems. Yet as noted above, many systems of practical interest are driven out of equilibrium and display (indeed, rely upon) nonlinear behaviours. Recently a body of work has emerged focusing on this active nonlinear microrheology regime (Habdas et al. 2004; Meyer et al. 2005; Squires \& Brady 2005; Khair \& Brady 2006; Wilson et al. 2009). In such a system, tracer particles undergo displacements not only due to random thermal fluctuations, but also due to the application of an external force applied directly to the tracer, or 'probe'. The dispersion is driven out of equilibrium, and as with macrorheology, dynamic responses such as viscosity can be measured. Since the tracer interrogates the material at its own (micro)scale, much smaller samples are required compared to traditional macrorheology, and localized material heterogeneity can be explored. This is a particular benefit for rare biological materials and small systems such as cells. Khair \& Brady (2006) recently established the theory that predicts the microviscosity of dilute systems of colloids, and defined the relationship between micro- and macroviscosity - a critical step in the development of microrheology as an experimental tool. Recent experiments confirm the theory (and raise additional questions) (Meyer et al. 2005; Squires 2008; Wilson et al. 2009).

But in both theory and experiment, the focus thus far has been on the mean response of the material - the viscosity - and far less work has been devoted to particle fluctuations that occur due to collisions between the probe and bath particles. As the probe moves through the dispersion it must push neighbouring particles out of its way; these collisions induce fluctuations in the probe's velocity, scattering it from its mean path. Is this scattering diffusive? Is it isotropic? How important is the scattering compared to the Brownian diffusion the probe simultaneously undergoes? The answers to these questions are fundamental to understanding the motion of an active microscale particle - important for both scientific and technology considerations. Little work has been published on this topic (Habdas et al. 2004), even though it has major implications for a wide range of technologies beyond material interrogation.

Previous study of particle fluctuations in colloidal macrorheology shows that imposing a shearing flow on a suspension enhances particle diffusivity (Leighton \& Acrivos 1987; Morris \& Brady 1996; Brady \& Morris 1997; Breedveld et al. 1998). The imposed shear flow drives the microstructure from equilibrium, giving rise to mechanisms of diffusion not present in a quiescent suspension: a deformed microstructure and interparticle collisions. A forced microrheological probe also imposes a flow that drives the suspension from equilibrium, again leading to a 

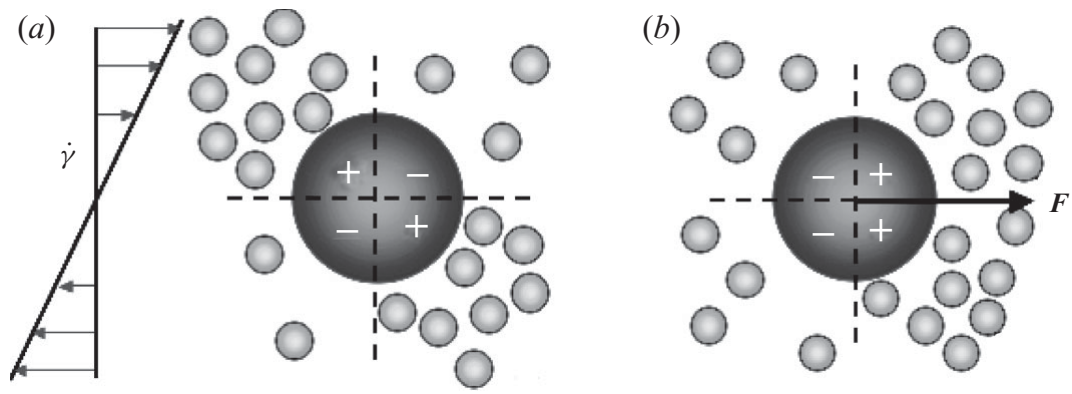

FIGURE 1. Microstructural deformation under $(a)$ macrorheological shear flow and $(b)$ microrheological forcing. In simple shearing motion $(a)$, bath particles accumulate along the compressional axis and deplete along the extensional axis. A tracer at the origin (the dark sphere) experiences both in-plane and out-of-plane bath particle gradients. In $(b)$, the (dark) tracer itself deforms the microstructure, accumulating particles on its upstream face and leaving a wake of depletion behind, creating an axisymmetric structure with only longitudinal and transverse components.

deformed microstructure and interparticle collisions - and hence to an analogous force-induced diffusion, or 'microdiffusivity'. It is the primary objective of this study to extend the theoretical model of active nonlinear microrheology to one that is explicit in the fluctuations of the microstructure, and thereby develop expressions for predicting the resultant force-induced diffusion.

It is also useful to ask whether the qualitative agreement between micro- and macroviscosity can be extended to the micro- and macrodiffusivity. Both the shearand force-induced diffusions grow out of fundamentally similar mechanisms: external forcing causes the tracer to scatter off of the microstructure, rather than wander passively through it. But the directionality and magnitude of the scattering depend on the shape of the deformed microstructure, and this asymmetry is distinct for the two cases, as illustrated in figure 1. The idea of whether a direct correlation between macro shear-induced diffusion and micro force-induced diffusion is possible (or even necessary) will be explored in this study, and a comparison is sought between the two.

To build up a physical model, we follow the example of Squires \& Brady (2005) and consider the motion of a Brownian probe driven by an externally applied force through a dispersion of neutrally buoyant-force- and torque-free colloidal particles. The size ratio of probe to bath particle is arbitrary. As the probe particle moves through the suspension it must push neighbouring particles out of its way; a buildup of background particle concentration forms in front of the advancing probe and a deficit or wake trails it. The bath particles (including the probe) undergo Brownian diffusion due to thermal fluctuations of the solvent, which acts to restore the deformed microstructure to an equilibrium configuration. The ratio of advective forcing to entropic restoring force is the Péclet number, $P e=F^{e x t} /(k T / b)$, where $k T$ is the thermal energy and $b$ the bath particle size. In the linear-response regime $(P e \rightarrow 0)$, diffusion of the bath particles dominates. As the probe forcing increases and $P e$ grows beyond $\sim O(1)$ - the active nonlinear-response regime - advection plays an increasingly important role in the shape of the distorted microstructure. Since the details of this shape govern the strength and likelihood of collisions between probe and bath particles, its determination is critical to understanding the resultant force-induced diffusive spread of the probe's trajectory. 
The spatiotemporal distribution of bath particles obeys a Smoluchowski equation. Prior treatments of active microrheology formulated the expression for the steady microstructure moving relative to a fixed probe. We extend this approach by considering both the steady microstructure deformation and fluctuations in the microstructure responsible for diffusion of the probe. The resulting Smoluchowski equations are solved for all $P e$ by a combination of perturbation methods and numerical computation to obtain both the steady microstructure along with a new quantity, the probability-weighted collisional displacements of the probe relative to the bath particles.

Scaling arguments are useful for predicting the behaviour for extreme values of the Péclet number. For random-walk processes, the diffusivity scales as

$$
D \sim \frac{l^{2}}{\tau}
$$

where $l$ is the size of a probe step and $\tau$ is the decorrelation time. For very weak forcing, $P e \ll 1$, Brownian diffusion dominates the motion; so the time scale is $\tau \sim(a+b)^{2} / D_{a}$, where $D_{a}$ is the self-diffusivity of an isolated probe particle of size $a$. In this linear-response regime, $l \sim P e(a+b)$. The number of diffusive steps depends on the number of bath particle collisions; thus, for very small $P e$, the microdiffusivity should scale quadratically in the forcing and linearly in volume fraction of bath particles $\phi_{b}$ :

$$
D^{\text {micro }} \sim P e^{2} \phi_{b} D_{a}, \quad P e \ll 1 .
$$

For large forcing, $P e \gg 1$, the time scale is now advective, $\tau \sim(a+b) / U$, the probe can move $l \sim(a+b)$ in that time and the force-induced microdiffusivity should scale linearly with the Péclet number and in volume fraction of bath particles:

$$
D^{\text {micro }} \sim P e \phi_{b} D_{a}, \quad P e \gg 1 .
$$

In the remaining sections of this paper, we propose and examine an extended model for active nonlinear microrheology that is explicit in the probe fluctuations, and explore the resultant force-induced diffusion. In $\S 2$, we formulate the Smoluchowski equation that governs the evolution of the microstructure in physical space, along with kinematic expressions for the probe flux. The latter comprises advective and interparticle contributions, and from these the diffusive flux of the probe is extracted and separated into Brownian and flow-induced components. To make analytical progress an assumption of diluteness is made. In $\S 3$ the Smoluchowski equation is separated into steady and fluctuating components, completing the formulation of the problem. In $\S 4.1$ the case of asymptotically weak probe forcing, $P e \ll 1$, is studied. Regular perturbation expansions are sufficient to obtain the $O\left(\phi_{b}\right)$ correction to the long-time self-diffusivity, which corresponds to passive diffusion: $D \sim D_{a}\left(1-2 \phi_{b}\right)$, where $\phi_{b}$ is the (dilute) volume fraction of bath particles and $D_{a}$ is the diffusivity of an isolated probe (Batchelor 1976). Higher orders in $\phi_{b} P e$ are required in order to find the first effect of the forcing on particle self-diffusion and we proceed to $O\left(\phi_{b} P e^{2}\right)$ whereupon the problem becomes singular, which requires the use of matched asymptotic expansions. In $\S 4.2$ we shift focus to the opposite extreme of very strong probe forcing, or $P e \gg 1$, and the nonlinear response of the microstructure is exposed. This limit is also singular with a boundary layer at the probe surface. To solve for probe fluctuations at arbitrary values of the Péclet number, a numerical solution is required, and a finite difference scheme is employed to this end in $\S 4.3$. In $\S 5$ we present an alternative solution of the problem based upon measurements of 
the probe's displacements obtained by Brownian dynamics simulation, presented juxtaposed to the Smoluchowski results. Section 6 is devoted to a comparison of microdiffusivity to the macrodiffusivity (theory and experiments). Throughout, we consider a simplified model that neglects hydrodynamic interactions between particles; this simplification affords insight into the basic physics of diffusive behaviour arising from interparticle forcing, but could be extended to include other interparticle forces such as hydrodynamic interactions; the approach also offers a direct solution to related problems such as self-propelled objects. The study is concluded with a discussion in $\S 7$, including a brief discussion of hydrodynamic interactions, self-propulsion and non-spherical shapes.

\section{Microdiffusivity}

The theoretical framework of active microrheology begins with a simple model: a single Brownian probe particle of radius $a$ is dragged by a constant external force $\boldsymbol{F}^{\text {ext }}$ through a dispersion of colloidal particles, all of size $b$, which are immersed in a solvent of density $\rho$ and viscosity $\eta$. The importance of fluid inertia relative to the viscous shearing forces is characterized by the Reynolds number, $R e=\rho U a / \eta$, where $U$ is the characteristic velocity of the moving probe, and for micrometre-sized probes $R e \ll 1$, so that the fluid mechanics are governed by Stokes flow. The advective forcing of the probe acts to deform the microstructure of the bath, while the Brownian motion of the bath particles counteracts it in an attempt to restore equilibrium. This interplay drives fluctuations in the probe's velocity that give rise to diffusive behaviour. The primary goals of this section are to examine the dispersive contributions to the probe's flux relative to the bath, formulate the expression for the force-induced component and show that the force-induced dispersive motion is indeed diffusive.

We begin by defining the hard-sphere model for the interactive potential $V(r)$ between a particle of size $a$ and a particle of size $b$ located at positions $\boldsymbol{x}_{1}$ and $\boldsymbol{x}_{2}$, respectively; they are separated by $\boldsymbol{r}=\boldsymbol{x}_{2}-\boldsymbol{x}_{1}$. Thus defined, the particles exert no force on each other until their surfaces touch, $r=a+b$, at which point an infinite repulsive potential is exerted to prevent their overlap:

$$
V(r)= \begin{cases}\infty, & r<a+b, \\ 0, & r>a+b .\end{cases}
$$

In general, the radii $a$ and $b$ at which particles exert the hard-sphere force are not the same as their hydrodynamic radii, $a_{h}$ and $b_{h}$, the surface at which the no-slip boundary condition is obeyed. Various physical conditions of the colloids or the solvent can extend the effective size of the particle beyond the hydrodynamic radius, e.g. steric hindrance or an ionic screening layer; two particles may then experience a hard-sphere repulsive force at overlap of their increased effective or 'thermodynamic' radii.

Following the excluded annulus model of Morris \& Brady (1996) and Bergenholtz et al. (2002), the ratios $\lambda_{a}=a / a_{h}$ and $\lambda_{b}=b / b_{h}$ give the relative importance of hydrodynamic interactions between particles (figure 2). When $\lambda_{a}, \lambda_{b} \sim O(1)$, hydrodynamic interactions strongly affect the particle configuration; as $\lambda_{a}, \lambda_{b} \rightarrow 1$, the particles get close enough that short-range lubrication forces become important. We shall assume a system of particles for which $\lambda_{a}, \lambda_{b} \gg 1$, where hydrodynamic interactions are negligible compared to interparticle and thermal forces. This model captures the essential features of the dispersive process while keeping the analyses 


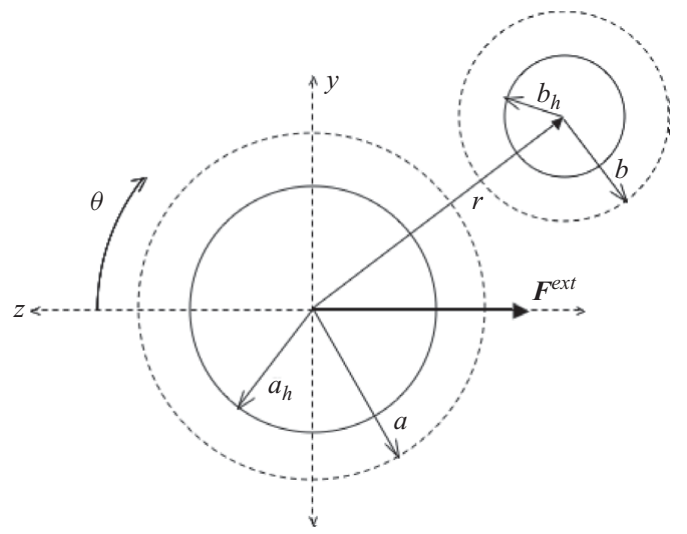

Figure 2. Pair interaction for the probe and bath particle.

as simple as possible; the effect of hydrodynamic interactions are discussed briefly in $\S 7$.

We consider the probe amid a dispersion of $N-1$ bath particles in a configuration $\boldsymbol{x}_{N}$. The distribution of particles is given by an $N$-particle probability density, $P_{N}\left(\boldsymbol{x}_{1}, \boldsymbol{x}_{2}, \ldots, \boldsymbol{x}_{N} ; t\right)$, which obeys a Smoluchowski equation

$$
\frac{\partial P_{N}}{\partial t}+\sum_{i=1}^{N} \nabla_{i} \cdot \boldsymbol{j}_{i}=0
$$

where the sum is over all particles in the dispersion, and the flux of particle $i$ is given by

$$
\boldsymbol{j}_{i}=\boldsymbol{U}_{i} P_{N}\left(\boldsymbol{x}_{N} ; t\right)-\sum_{j=1}^{N} \boldsymbol{D}_{i j} \cdot \nabla_{j}\left(\ln P_{N}\left(\boldsymbol{x}_{N} ; t\right)+V_{N} / k T\right) P_{N}\left(\boldsymbol{x}_{N} ; t\right) .
$$

Here, $k T$ is the thermal energy, $\boldsymbol{D}_{i j}=k T \boldsymbol{M}_{i j}$ is the relative Brownian diffusivity between particles $i$ and $j$ and $\boldsymbol{M}_{i j}$ is the mobility tensor relating the velocity of particle $i$ to the force exerted on particle $j$. The first term on the right-hand side of (2.3) is the advective flux of particle $i$ with velocity $\boldsymbol{U}_{i}$, the second term is the flux due to entropic gradients in the microstructure and $\boldsymbol{D}_{i j} \cdot\left(\nabla_{j} V_{N} / k T\right) P_{N}$ gives the flux of particle $i$ due to the forcing of interactive potential with particle $j$.

In the dilute limit, only pair interactions are important, and the probability $P_{N}$ in (2.2) and (2.3) reduces to the pair probability of finding the probe at position $\boldsymbol{x}_{1}$ and a bath particle at position $\boldsymbol{x}_{2}$. To analyse the relative flux between probe and bath, it is convenient to change to a frame of reference moving with the probe, placing the probe at $\boldsymbol{z}=\boldsymbol{x}_{1}$, and a bath particle at $\boldsymbol{r}=\boldsymbol{x}_{2}-\boldsymbol{x}_{1}$. The pair Smoluchowski equation becomes

$$
\frac{\partial P_{2}(\boldsymbol{z}, \boldsymbol{r} ; t)}{\partial t}+\nabla_{z} \cdot \boldsymbol{j}_{a}+\nabla_{r} \cdot\left(\boldsymbol{j}_{b}-\boldsymbol{j}_{a}\right)=0
$$

Here, the subscripts $a$ and $b$ refer to the probe and the bath particle, respectively. We are interested in the flux of the probe relative to any other particle, and so we integrate $P_{2}(\boldsymbol{z}, \boldsymbol{r} ; t)$ over all possible positions of a bath particle, to obtain the single-particle Smoluchowski equation for the probe particle. Applying the divergence theorem and 
noting that relative flux is zero at contact and at infinity, we have

$$
\frac{\partial P_{1}(\boldsymbol{z} ; t)}{\partial t}+\nabla_{z} \cdot\left\langle\boldsymbol{j}_{a}\right\rangle=0
$$

where $\left\langle\boldsymbol{j}_{a}\right\rangle \equiv \int \boldsymbol{j}_{a} \mathrm{~d} \boldsymbol{r}$. The unbounded domain of the probe makes a transformation

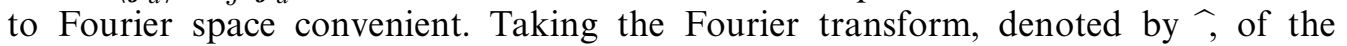
Smoluchowski equation (2.5) yields

$$
\frac{\partial \hat{P}_{1}(\boldsymbol{k} ; t)}{\partial t}+\mathrm{i} \boldsymbol{k} \cdot\left\langle\hat{\boldsymbol{j}}_{a}\right\rangle=0
$$

where the average flux $\left\langle\hat{\boldsymbol{j}}_{a}\right\rangle$ from (2.3) has also been transformed to Fourier space:

$$
\left\langle\hat{\boldsymbol{j}}_{a}\right\rangle=\left(\boldsymbol{U}_{a}-\mathrm{i} \boldsymbol{k} D_{a}\right) \hat{P}_{1}(\boldsymbol{k} ; t)+D_{a} \int \nabla_{r} \hat{P}_{2}(\boldsymbol{k}, \boldsymbol{r} ; t) \mathrm{d} \boldsymbol{r} .
$$

Here, $\boldsymbol{U}_{a}=\boldsymbol{F}^{\text {ext }} / 6 \pi \eta a$ is the probe velocity due to the imposed constant external force, $D_{11} \equiv D_{a}$ the probe self-diffusivity, $\mathrm{i}$ the imaginary unit and the angle brackets \langle\rangle denote an ensemble average over all possible suspension configurations. $\hat{P}_{2}(\boldsymbol{k}, \boldsymbol{r} ; t)$ and $\hat{P}_{1}(\boldsymbol{k} ; t)$ are the Fourier transforms of $P_{2}(\boldsymbol{z}, \boldsymbol{r} ; t)$ and $P_{1}(\boldsymbol{z} ; t)$, respectively.

The last term in (2.7) explicitly preserves the effect of bath particles on the probe. In order to determine the average probe flux, the distribution of the bath relative to the probe must be determined. To this end we define the structure function $g(\boldsymbol{k}, \boldsymbol{r} t)$ :

$$
\hat{P}_{2}(\boldsymbol{k}, \boldsymbol{r} ; t) \equiv n_{b} g(\boldsymbol{k}, \boldsymbol{r} ; t) \hat{P}_{1}(\boldsymbol{k} ; t),
$$

where $n_{b}$ is the undisturbed number density of bath particles far from the probe.

Expression (2.8) is similar to the familiar definition of the pair-distribution function in physical space, $P_{2}(\boldsymbol{z}, \boldsymbol{r} ; t)=n_{a} n_{b} g(\boldsymbol{z}, \boldsymbol{r} ; t)$ (since $\boldsymbol{z}$ is the origin, it is typically omitted). But $g(\boldsymbol{k}, \boldsymbol{r} ; t)$ is not simply the Fourier transform of $g(\boldsymbol{z}, \boldsymbol{r} ; t)$. Rather, we have defined in Fourier space the microstructure $g(\boldsymbol{k}, \boldsymbol{r} ; t)$ where the $\boldsymbol{k}$-dependence explicitly preserves fluctuations of the probe relative to the origin.

Also note that $g(\boldsymbol{k}, \boldsymbol{r}, t)$ is not to be confused with the structure factor, $S(z, \boldsymbol{q}, t)=\mathscr{F}_{r}\left[P_{2}(z, \boldsymbol{r}, t)\right]$, corresponding to a Fourier transform with respect to the separation vector $\boldsymbol{r}$ between the probe and the bath particle. Indeed, we solve for the distribution of bath particles relative to the probe in physical (real) space $r$. The Fourier transform variable $\boldsymbol{k}$ is with respect to the absolute position of the probe, $z$. To determine the diffusive motion of the probe one imagines a concentration gradient of a dilute collection of probes; these physical-space gradients correspond to algebraic multiples of the wave vector, $\boldsymbol{k}$, in Fourier space. The probe flux down this concentration gradient is influenced by the interaction with bath particles distributed according to $g(\boldsymbol{k}, \boldsymbol{r}, t)$, a distribution that must be determined for all $\boldsymbol{r}$. flux:

Combining (2.7) and (2.8) we obtain the following for the steady average probe

$$
\left\langle\hat{\boldsymbol{j}}_{a}\right\rangle=\left[\boldsymbol{U}_{a}-D_{a} \mathrm{i} \boldsymbol{k}+n_{b} D_{a} \int \nabla_{r} g(\boldsymbol{k}, \boldsymbol{r}) \mathrm{d} \boldsymbol{r}\right] \hat{P}_{1}(\boldsymbol{k}) .
$$

For the long-time self-diffusion of the probe, we consider the short wave vector (long length scale) limit and expand $g(\boldsymbol{k}, \boldsymbol{r})$ for small $\boldsymbol{k}$, corresponding to a weak gradient in the 'concentration of probes':

$$
g(\boldsymbol{k}, \boldsymbol{r})=g_{0}(\boldsymbol{r})+\mathrm{i} \boldsymbol{k} \cdot \boldsymbol{d}(\boldsymbol{r})+\cdots,
$$


which immediately yields the two terms governing the scattering of the probe: the steady microstructure, $g_{0}(\boldsymbol{r})$, and the probability-weighted displacement of the probe, $\boldsymbol{d}(\boldsymbol{r})$ - i.e. the likelihood of a collision and the strength and direction of the probe displacement upon collision, respectively. Substituting this into the expression for probe flux yields

$$
\left\langle\hat{\boldsymbol{j}}_{a}\right\rangle=\left(\left[\boldsymbol{U}_{a}+n_{b} D_{a} \int \nabla g_{0}(\boldsymbol{r}) \mathrm{d} \boldsymbol{r}\right]-D_{a} \mathrm{i} \boldsymbol{k} \cdot\left[\boldsymbol{I}-n_{b} \int \nabla_{r} \boldsymbol{d}(\boldsymbol{r}) \mathrm{d} \boldsymbol{r}\right]\right) \hat{P}_{1}(\boldsymbol{k}),
$$

where $I$ is the identity tensor. Examination of (2.11) reveals the effect of the bath particles on both the mean $(O(1))$ and fluctuating $(O(\boldsymbol{k}))$ response of the overall suspension. The first bracketed term gives the probe's average speed through the fluid: $\boldsymbol{U}_{a}$ is reduced by the entropic reactive force of the microstructure, as given by the integral term. This reduction in probe speed due to the suspended particles was used by Squires \& Brady (2005) to define the microviscosity. Recalling that $i k$ terms represent diffusion, the second bracketed group gives the effective diffusivity of the probe; the third term its free Brownian diffusion, plus an increment due to interactions with the bath. This increment corresponds to hard-sphere interactions between the probe and bath that scatter the probe's mean path. From a phenomenological perspective, the effect of the bath particles is to reduce the mean velocity of the probe and increase the diffusive spread of its trajectory - the effective diffusivity. Defining the second bracketed group as the effective diffusivity of the probe and integrating by parts we obtain

$$
\boldsymbol{D}^{e f f} \equiv D_{a}\left[\boldsymbol{I}-n_{b} \oint_{r=a+b} \boldsymbol{n d} \mathrm{d} S\right]
$$

where $\boldsymbol{n}$ is the unit surface normal pointing outwards from the probe.

In the limit $P e \rightarrow 0$, the entropically hindered diffusion of a particle in a dilute suspension without hydrodynamic interactions must be recovered, $D^{\text {eff }}=D_{\infty}^{s}=$ $D_{a}\left(1-2 \phi_{b}\right)$ (Batchelor 1976), for $a / b=1$. Motivated by this fact, we denote the corresponding displacement field for the unforced probe $\boldsymbol{d}_{\text {entropic }} \equiv \boldsymbol{d}(P e=0)$. Hence, we express the total displacement field as a sum of entropic and mechanical contributions:

$$
\boldsymbol{d}=\boldsymbol{d}_{\text {entropic }}+\boldsymbol{d}^{\prime}
$$

where $\boldsymbol{d}^{\prime}$ is the probe fluctuation over and above that for hindered passive diffusion. As we show below, $\boldsymbol{d}_{\text {entropic }}$ contributes $-2 \phi_{b} D_{a}$ to the effective diffusivity; so we write

$$
\boldsymbol{D}^{\text {eff }}=D_{a} \boldsymbol{I}\left(1-2 \phi_{b}\right)+\boldsymbol{D}^{\text {micro }},
$$

where we have defined

$$
\boldsymbol{D}^{\text {micro }} \equiv n_{b} D_{a} \oint \boldsymbol{n} \boldsymbol{d}^{\prime} \mathrm{d} S .
$$

The effective diffusivity of a tracer particle is its bare diffusivity, $D_{a} \boldsymbol{I}$, minus the entropic hindrance of the bath, $2 \phi_{b} D_{a} I$, plus an enhancement due to mechanical scattering by the other bath particles, $\boldsymbol{D}^{\text {micro }}$. The force-induced microdiffusivity is proportional to the number density of bath particles, the isolated probe self-diffusivity, and to the first moment of the hard-sphere deflections.

It remains only to obtain the steady microstructure $g_{0}(\boldsymbol{r})$ and the first fluctuation correction, $\boldsymbol{d}^{\prime}(\boldsymbol{r})$. 


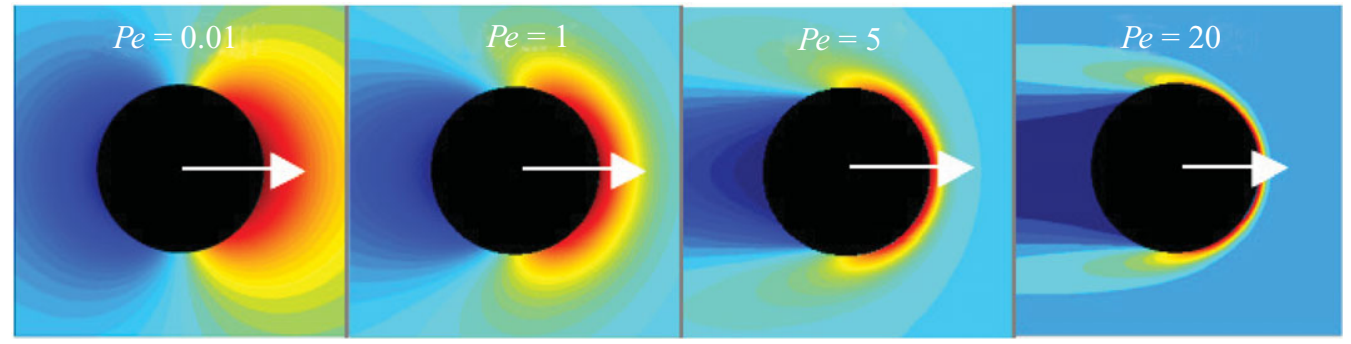

FIGURE 3. (Colour online) Theoretical predictions for the deformed microstructure around a moving probe particle in the absence of hydrodynamic interactions at the pair level. The test particle is moving to the right and there is a build-up of background particle density in front (red) of the probe and a deficit (dark blue) in the trailing wake (Squires \& Brady 2005).

\section{Non-equilibrium microstructure}

The goal of this section is to formulate an expression governing the evolution of the microstructure $g(\boldsymbol{k}, \boldsymbol{r})$. The Smoluchowski equation governing the pair probability in Fourier space gives the evolution of the fluctuating microstructure:

$$
\frac{\partial \hat{P}_{2}}{\partial t}+\nabla_{r} \cdot\left[\boldsymbol{U}_{r}-D_{r} \nabla_{r}\right] \hat{P}_{2}+D_{a} \mathrm{i} \boldsymbol{k} \cdot \nabla_{r} \hat{P}_{2}+\mathrm{i} \boldsymbol{k} \cdot \hat{\boldsymbol{j}}_{a}=0
$$

where we have defined the relative Brownian diffusivity between the probe and the bath particle, $D_{r} \equiv D_{a}+D_{b}$ and $U_{r} \equiv U_{a}-U_{b}$. With the definition of $g(\boldsymbol{k}, \boldsymbol{r})$ in (2.8) and substituting (2.6) into (3.1) we have leading order in diluteness, at steady state:

$$
\begin{array}{rlrl}
\nabla_{r} \cdot\left[\boldsymbol{U}_{r}-D_{r} \nabla_{r}\right] g+2 D_{a} \mathrm{i} \boldsymbol{k} \cdot \nabla_{r} g & =0, & \\
\boldsymbol{n} \cdot\left[\boldsymbol{U}_{r} g-D_{r} \nabla_{r} g+D_{a} \mathrm{i} \boldsymbol{k} g\right] & =0 & & \text { at } r=a+b, \\
g & \sim 1 & \text { as } r \rightarrow \infty,
\end{array}
$$

in which conservation requires a no-flux boundary condition at contact and there is no long-range order. The equations are made dimensionless by scaling quantities as

$$
r \sim a+b, \quad U \sim F^{e x t} / 6 \pi \eta a, \quad D \sim D_{a}+D_{b}=\frac{k T}{6 \pi \eta}\left(\frac{1}{a}+\frac{1}{b}\right),
$$

and together with of the expansion (2.10) of $g(\boldsymbol{k}, \boldsymbol{r})$, the steady microstructure obeys

$$
\begin{aligned}
\nabla^{2} g_{0}-P e \boldsymbol{u} \cdot \nabla g_{0} & =0, \\
\boldsymbol{n} \cdot\left[\nabla g_{0}-P e \boldsymbol{u} g_{0}\right] & =0 \quad \text { at } r=1, \\
g_{0} & \sim 1 \quad \text { as } r \rightarrow \infty,
\end{aligned}
$$

where $\boldsymbol{u}$ is the unit vector parallel to probe forcing. Squires \& Brady (2005) have solved this problem analytically for all $P e=F^{e x t} /(k T / b)$. A contour plot in figure 3 shows the perturbed steady microstructure $g_{0}$ for a range of $P e$.

The expression governing the probability-weighted displacement also forms an advection-diffusion equation, but is forced by gradients in the steady microstructure:

$$
\begin{array}{rlrl}
\nabla^{2} \boldsymbol{d}-P e \boldsymbol{u} \cdot \nabla \boldsymbol{d} & =\beta \nabla g_{0}, & \\
\boldsymbol{n} \cdot(\nabla \boldsymbol{d}-P e \boldsymbol{u d}) & =\frac{1}{2} \beta g_{0} & & \text { at } r=1, \\
\boldsymbol{d} & \sim 0 & & \text { as } r \rightarrow \infty,
\end{array}
$$


where $\beta \equiv 2 /(1+a / b)$. Hence, the displacement field $\boldsymbol{d}$ is coupled to $g_{0}$. In the next section the coupled system is solved analytically in the limit of small and large $P e$, and numerically for arbitrary values of the Péclet number.

\section{Results}

\subsection{Low-Pe limit}

For small Péclet number, Brownian diffusion of the bath particles easily repairs the deformation of the microstructure caused by the probe's motion. Since the bath is hardly displaced from equilibrium, we approach the solution with a perturbation expansion in small Pe. Recalling (3.6)-(3.8), however, it is apparent that the problem is singular: at some distance $\rho \sim r P e$ from the probe, advection is as important as diffusion. The domain is divided into two regions, and matched asymptotic expansions yield the expression for the steady microstructure $g_{0}$ to $O\left(P e^{2}\right)$ :

$$
g_{0}(\boldsymbol{r} ; P e)=1-\frac{1}{2} \boldsymbol{u} \cdot \frac{\boldsymbol{r}}{r^{3}} P e+\frac{1}{4}\left(\frac{1}{r}-\frac{1}{3} \boldsymbol{u} \boldsymbol{u}:\left[\frac{\boldsymbol{I}}{r^{3}}-3 \frac{\boldsymbol{r} \boldsymbol{r}}{r^{5}}\right]-\boldsymbol{u u}: \frac{\boldsymbol{r} \boldsymbol{r}}{r^{3}}\right) P e^{2},
$$

which agrees with the solution that Squires \& Brady obtained to $O(P e)$, which we have extended here to $O\left(P e^{2}\right)$.

A similar method is applied to (3.9)-(3.11) to obtain the fluctuation field. The $P e^{0}$ term of the expansion of $\boldsymbol{d}$ yields the solution

$$
\boldsymbol{d}_{\text {entropic }} \equiv \boldsymbol{d}^{(0)}=-\frac{1}{4} \beta \frac{\boldsymbol{r}}{r^{3}},
$$

which gives

$$
D^{e f f}(P e=0)=D_{a}\left[1-\frac{1}{2}(1+a / b)^{2} \phi_{b}\right],
$$

which, for equal probe and bath particle size, recovers the long-time self-diffusivity of an isolated sphere in a quiescent solvent reported by Batchelor (1976), $D^{e f f}(P e=0)=D_{\infty}^{s}=D_{a}\left(1-2 \phi_{b}\right)$. Since there is no flow at $P e=0$, this $O\left(\phi_{b}\right)$ correction is due to the entropic hindrance of the bath.

It is interesting to note that the $O(1)$ solution for $\boldsymbol{d}$ is the same as the $O(P e)$ solution for $g_{0}$. In fact the problems for $g_{0}$ and $\boldsymbol{d}$ are identical in the limit $P e \rightarrow 0$. In the linear-response regime, whether the forcing is by external means, $g_{0}$, or by thermal fluctuation, $\boldsymbol{d}$, the resulting mobility reduction or diffusivity $-k T$ times the mobility - is the same.

At the next order in $P e$, we resolve the vector $\boldsymbol{d}$ into scalar components parallel and transverse to the direction of the probe's velocity, $\boldsymbol{d}_{\|}=d_{z} \boldsymbol{u}, \boldsymbol{d}_{\perp, x}=d_{x} \boldsymbol{e}_{x}$ and $\boldsymbol{d}_{\perp, y}=d_{y} \boldsymbol{e}_{y}$. The $O\left(\phi_{b} P e\right)$ fluctuation makes no contribution to the microdiffusivity in keeping with scaling predictions, since $\boldsymbol{n d}(1) \sim \boldsymbol{u} \cdot \boldsymbol{n n} \boldsymbol{n}$ and the displacement of a sphere has no coupling to odd tensors.

Proceeding to the next order in $P e$, the singular nature of the problem becomes evident, as the solution by regular perturbation expansion fails to decay to zero far from the probe. An asymptotic expansion in the inner region is matched to the solution in the outer region, giving the proper far-field condition for the inner solution. The first correction to the fluctuation that gives rise to microdiffusive 
behaviour is then

$$
\begin{aligned}
d_{\|}^{(2)}= & \beta\left[-\frac{13}{48}-\left(\frac{67}{360} \frac{\boldsymbol{r}}{r^{3}}-\frac{11}{480}\left[3 \frac{\boldsymbol{r}}{r^{5}}-5 \boldsymbol{u} \boldsymbol{u}: \frac{\boldsymbol{r r} \boldsymbol{r}}{r^{7}}\right]\right) \cdot \boldsymbol{u}\right] \\
& -\beta\left[\frac{13}{144}\left(\left[\frac{\boldsymbol{r}}{\boldsymbol{r}^{3}}-3 \boldsymbol{u u}: \frac{\boldsymbol{r} \boldsymbol{r}}{r^{5}}\right]-\frac{1}{96}\left(41 \frac{\boldsymbol{r}}{r}-15 \boldsymbol{u} \boldsymbol{u}: \frac{\boldsymbol{r} \boldsymbol{r} \boldsymbol{r}}{r^{3}}\right)\right) \cdot \boldsymbol{u}\right], \\
d_{\perp}^{(2)}= & \beta\left\{-\frac{7}{80 r^{2}}+\frac{13}{48} \boldsymbol{u} \boldsymbol{u}: \frac{\boldsymbol{r} \boldsymbol{r}}{r^{4}}+\frac{11}{480}\left[\frac{1}{r^{4}}-5 \boldsymbol{u} \boldsymbol{u}: \frac{\boldsymbol{r} \boldsymbol{r}}{r^{6}}\right]+\frac{5}{32}[1-\boldsymbol{u u}: \boldsymbol{r} \boldsymbol{r}] \boldsymbol{r} \cdot \boldsymbol{e}_{y}\right\},
\end{aligned}
$$

which yields for the microdiffusivity in the limit of $P e \ll 1$ (plotted in figure 6):

$$
\begin{aligned}
& D_{\|}^{\text {micro }}=\frac{79}{180}\left(1+\frac{a}{b}\right)^{2} D_{a} P e^{2} \phi_{b}, \\
& D_{\perp}^{\text {micro }}=\frac{11}{60}\left(1+\frac{a}{b}\right)^{2} D_{a} P e^{2} \phi_{b} .
\end{aligned}
$$

When forced very weakly through a dilute suspension, a probe particle diffuses with its bare diffusivity $D_{a}$ minus an entropic hindrance due to the presence of the bath particles that scales as $\phi_{b} D_{a}$ plus an enhancement due to hard-sphere collisions with the bath particles - characteristic of the Taylor dispersion for particles in a bulk flow. As predicted by scaling arguments in $\S 1$, in the low-Pe limit, the force-induced enhancement to the diffusion is quadratic in the forcing; it is also linear in the volume fraction of bath particles, $\phi_{b}$, and anisotropic, preferentially diffusing along the direction of forcing (as compared to the transverse direction) by a factor of 2.39 .

\subsection{High-Pe limit}

For very large Péclet number, the shape of the microstructure in front of the probe is deformed into two distinct regions: an outer region in which advection dominates diffusion and the microstructure is undisturbed, and an inner region - a $1 / P e$-thin boundary layer that forms on the upstream face of the probe - where diffusion balances advection. A Pe-long wake of particle deficit forms behind the probe, where probability for a probe/bath particle collision is small. The particles that reside inside the boundary layer provide the most probability for a strong hard-sphere deflection of the probe (cf. figure 3 ). Inside the boundary layer, a coordinate rescaling $R=(r-1) P e \sim O(1)$ preserves the diffusive term, properly reflecting the physics of the inner region and allowing satisfaction of the no-flux condition at contact. A singular perturbation expansion in powers of $P e^{-1}$ then obtains the deflection field in the boundary layer on the upstream face of the probe, $\pi / 2 \leqslant \theta \leqslant \pi$ :

$$
\begin{aligned}
d_{\|} & =-\frac{\beta}{12} \frac{\boldsymbol{u} \cdot \boldsymbol{n}}{\left(\boldsymbol{u} \cdot \boldsymbol{e}_{\theta}\right)^{2}}\left(1+(\boldsymbol{u} \cdot \boldsymbol{n})^{3}\right) \mathrm{e}^{P e(r-1) u \cdot \boldsymbol{n}} P e+O(1), \\
d_{\perp} & =-\frac{\beta}{12}(\boldsymbol{u} \cdot \boldsymbol{n})\left(\boldsymbol{u} \cdot \boldsymbol{e}_{\theta}\right)\left(\boldsymbol{n} \cdot \boldsymbol{e}_{y}\right) \mathrm{e}^{P e(r-1) \boldsymbol{u} \cdot \boldsymbol{n}} P e+O(1),
\end{aligned}
$$

where $\boldsymbol{u} \cdot \boldsymbol{n} \leqslant 0, \theta$ is the angle between $\boldsymbol{u}$ and the normal $\boldsymbol{n}$ and $\boldsymbol{e}_{\theta}$ is a unit vector in the direction of $\theta$. As expected from earlier scaling arguments, the microdiffusivity is linear in the forcing when $P e \gg 1$ :

$$
\begin{aligned}
& D_{\|}^{\text {micro }}=\frac{1}{4}\left(1+\frac{a}{b}\right)^{2}\left(\ln 2-\frac{1}{4}\right) D_{a} \text { Pe } \phi_{b}+O(1), \\
& D_{\perp}^{\text {micro }}=\frac{1}{32}\left(1+\frac{a}{b}\right)^{2} D_{a} \text { Pe } \phi_{b}+O(1) .
\end{aligned}
$$




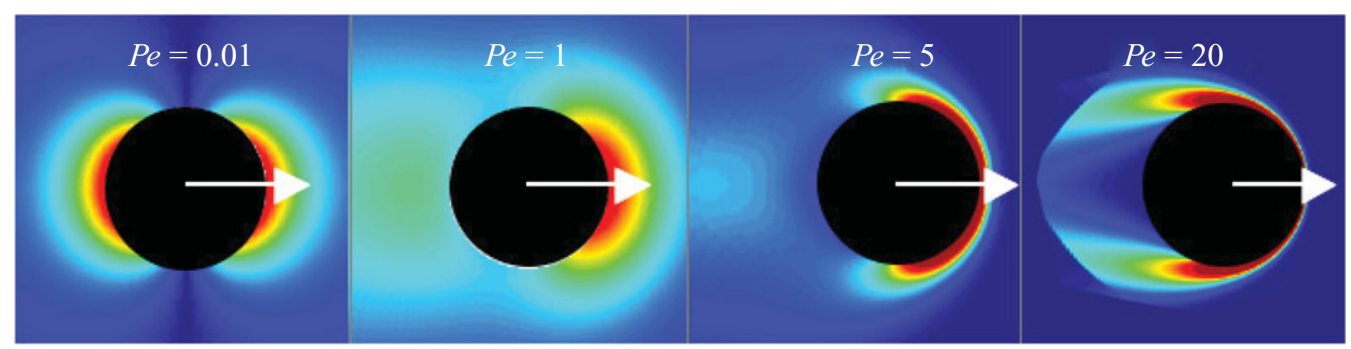

FIgURE 4. (Colour online) The fluctuation field longitudinal to probe forcing. Blue areas indicate regions of weak or no deflection; red areas indicate probability of strong deflection.

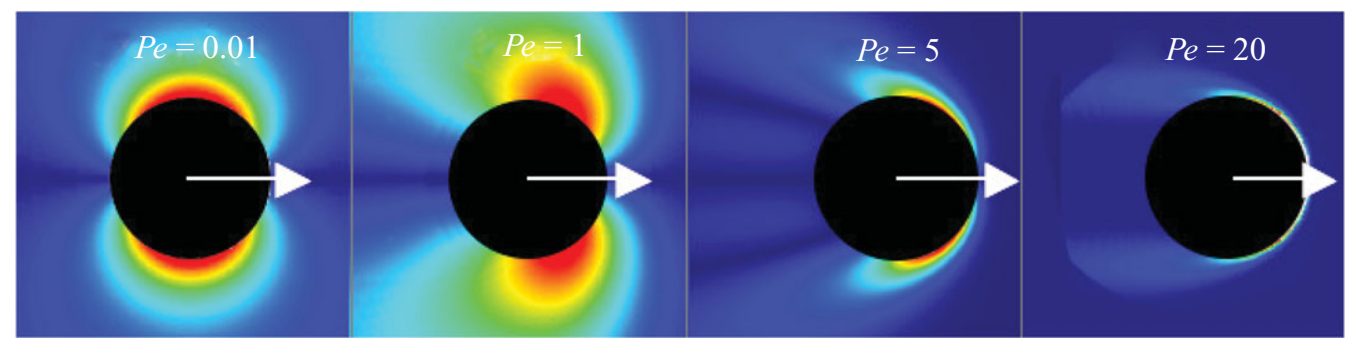

FIGURE 5. (Colour online) The fluctuation field transverse to probe forcing. Blue areas indicate regions of weak or no deflection; red areas indicate probability of strong deflection.

As in the low-Pe limit, the large $P e$ microdiffusivity is also transversely anisotropic, with a longitudinal-to-transverse preference of approximately $7 / 2$. The effect of the hard-sphere collisions is a $P e$-large diffusive scattering of the probe's trajectory.

The high- $P e$ analytical results are shown in figure 6 alongside those for small $P e$. When scaled with the volume fraction of bath particles and the probe's bare diffusivity $D_{a}$, the asymptotic limits of $P e \ll 1$ and $P e \gg 1$ form a framework to guide the analysis for intermediate values of the Péclet number, which is developed in the next section.

\subsection{Numerical solution for arbitrary $P e$}

To obtain the fluctuating microstructure over the full range of $P e$, a numerical solution of the full Smoluchowski equations (3.6)-(3.8) and (3.9)-(3.11) is required. The radial coordinate is rescaled with $P e^{-1}$ to obtain the stretched coordinate $R=P e(r-1)$. Because the flow is axisymmetric about the line of external forcing, derivatives of $g_{0}$ and $\boldsymbol{d}^{\prime}$ in the azimuthal angle are zero.

A central difference scheme is used to discretize gradients over the two-dimensional domain. Once a boundary layer forms, i.e. beyond $P e \gtrsim O(1)$, the radial gradients in the microstructure are almost entirely confined to the boundary layer. As $P e$ continues to grow and the boundary layer thins, a grid point concentration function that varies with $P e$ increases the density of grid points close to contact, yet retains sufficient resolution far from the probe to capture the physics throughout the upstream domain. The difference coefficients and operators for both radial and angular directions are compactly arranged into first- and second-order sparse matrices (Swaroop 2004). The solutions for the steady and fluctuating pair-distribution function are then obtained in MATLAB using a LAPACK iterative banded solver.

The steady microstructure is solved first (figure 3), and the gradients $\nabla g_{0}$ used to drive the fluctuation field $\boldsymbol{d}^{\prime}$. Contour plots for the deflection field are shown in figures 4 and 5; the anisotropy is evident. The longitudinal fluctuations show that at 


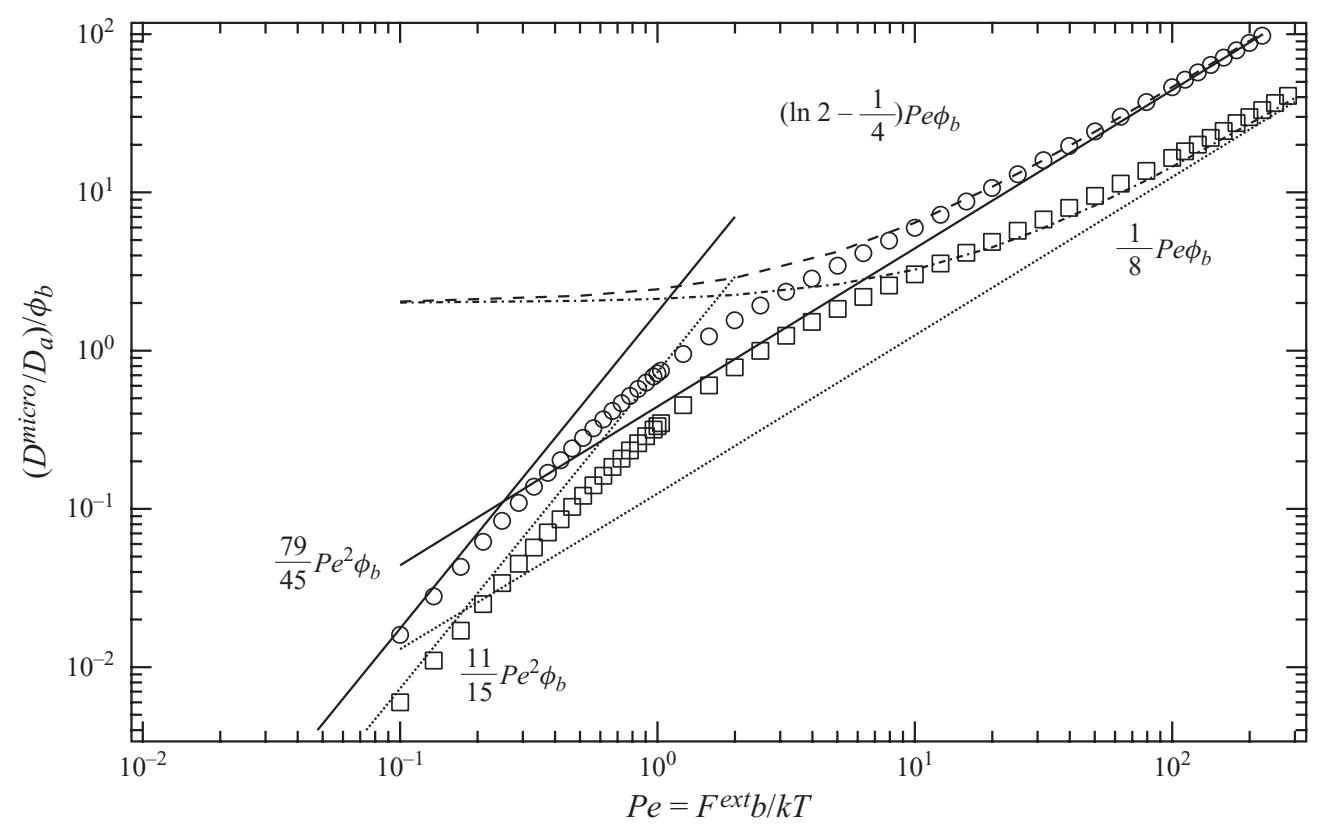

FIGURE 6 . The force-induced diffusivity $D^{\text {micro }}$, scaled with the probe bare diffusivity $D_{a}$ and the volume fraction of bath particles $\phi_{b}$. Analytical solutions for asymptotically small and large forcing are shown by solid lines (longitudinal) and dotted lines (transverse). Curved asymptotes exclude the entropic contribution (dashed for longitudinal; dash-dotted for transverse). Open symbols represent the numerical solution of the full Smoluchowski equation (circles for the longitudinal microdiffusivity, squares for the transverse microdiffusivity).

very small $P e$ (similar to $P e=0.01$ in the figure), the fluctuations form a dipole about the probe, with highest probability of a strong kick at the upstream face of the probe, and decaying as $\sim 1 / r^{2}$. As the Péclet number is increased, the boundary layer thins (similar to $P e=20$ in figure 4), and strong kicks to the probe result from particles swept into the boundary layer on the front of the probe. Particles diffuse and weakly advect around the probe, the boundary layer detaches and a wake forms behind it resulting in strong fore-aft asymmetry of probe fluctuations.

The transverse probe fluctuation field is shown in figure 5; for very weak forcing (similar to $P e=0.01$ in the figure), the region of highest probability for a strong deflection is at $\theta=\pi / 2$, with the distribution mirrored across the axis of symmetry. The resulting probe deflection is perpendicular to its mean motion. As $P e$ is increased, the probability of a lateral deflection is confined to the boundary layer.

The first moment of the fluctuation is numerically integrated over the surface of contact between the probe and the bath to obtain the microdiffusivity for a range of $0.01<P e<1000$, as shown in figure 6 . For very weak and very strong forcing, the numerical solution matches the analytical asymptotes.

Note that two sets of asymptotes are shown for $P e \gg 1$ in figure 6. The straight asymptotes correspond to the high-Pe microdiffusivity as given by (4.10) and (4.11). Recall that for the large- $P e$ analytical solution we found $\boldsymbol{d}$, the total fluctuation field, rather than that due to force-induced collisions only, $\boldsymbol{d}^{\prime}$ (cf. (2.13)). This is asymptotically correct for $\boldsymbol{D}^{\text {micro }}$ as $P e \rightarrow \infty$. But $\boldsymbol{D}^{\text {micro }}$ is defined in terms of $\boldsymbol{d}^{\prime}$ and, therefore, to be consistent, we should use $\boldsymbol{d}^{\prime}$ rather than $\boldsymbol{d}$-which gives the curved asymptote. For large values of the Péclet number the two coincide. 


\section{Solution via Brownian dynamics simulation}

The dynamics of probe and bath particle motion are governed by the Langevin equation, a force balance which includes Brownian, external, hydrodynamic and other interparticle forces. In the present case this equation reads

$$
\mathbf{0}=\boldsymbol{F}^{e x t}+\boldsymbol{F}^{B}+\boldsymbol{F}^{P},
$$

where the left-hand side is zero because inertia is not important in colloidal dispersions, and $\boldsymbol{F}^{e x t}=\mathbf{0}$ for all particles except the probe. A probe of size $a$ is placed among a randomly distributed bath of particles of size $b$. The external force is prescribed, and the other forces in (5.1) are given by

$$
\begin{array}{cc}
\overline{\boldsymbol{F}^{B}}=0 \quad & \overline{\boldsymbol{F}^{B}(0) \boldsymbol{F}^{B}(t)}=2 k T\left(6 \pi \eta a_{i}\right) \boldsymbol{I} \delta(t), \\
\boldsymbol{F}^{P}=\boldsymbol{F}^{H S} .
\end{array}
$$

Here, the overbar denotes a time average and $\delta(t)$ is the Dirac delta function; $a_{i} \equiv a$ for the probe and $a_{i} \equiv b$ for a bath particle. At each time step in the simulation the particle positions are updated with a Brownian step and in the case of the probe, an externally forced step. The hard-sphere displacement due to a collision between probe and bath particle is added next; since the hard-sphere force is singular - non-zero at contact only - special treatment is needed. To this end we use a modified 'potentialfree' algorithm (Heyes \& Melrose 1993; Carpen \& Brady 2005), in which overlaps resulting from the external and Brownian steps are corrected along the line of centres of the two particles, for a hard-sphere step $\Delta \boldsymbol{x}^{H S}$. For a complete description of Brownian dynamics of active microrheology, see Carpen \& Brady (2005).

A dilute bath can be achieved in two ways: first, a single bath particle and a single probe can be placed in the simulation cell and many, many simulations run in order to obtain a statistically large number of interparticle collisions. Alternatively, many 'ideal-gas' bath particles can be placed in the cell with one probe - i.e. only probe-bath particle collisions occur, and the bath particles simply pass through each other. Thus, an individual time step contains only one deterministic and one Brownian step - for each particle in the cell - but it could contain zero, one or several hardsphere displacements, depending on the number of bath particles within one step of contacting the probe. Since the bath particles do not directly see each other, they have no size except when they encounter the probe. It is their number concentration $n_{b}$, the contact length scale $(a+b)$ and the Brownian diffusivities $D_{a}$ and $D_{b}$ that govern the system dynamics. Varying the value of $\phi_{b}$ thus provides a means to compress the time required to obtain a sufficient number of collisions for statistical analysis, and should have no effect on probe diffusivity (although this turns out not to be precisely the case, as we show below). To this end, volume fractions of bath particles from $0.1 \leqslant \phi_{b} \leqslant 0.9$ were tested.

Simulations were conducted with values of the Péclet number ranging from 0.1 to 100 , volume fractions $\phi_{b}=0.1,0.3,0.5,0.7$ and 0.9 , and $a / b=1$. On average, a hard-sphere collision occurs during approximately $2 \%$ of the total number of time steps. Hence, to obtain sufficient resolution of the data, a set of 480 simulations of $10^{6}$ time steps each was run for each $P e$ and for each $\phi_{b}$. The overall displacement of the probe, $\boldsymbol{x}(t)$, was recorded at each time step.

The effective diffusivity of the probe, $\boldsymbol{D}^{\text {eff }}$, is obtained from the time rate of change of the mean-square displacement of the probe according to

$$
\boldsymbol{D}^{e f f}(t)=\frac{1}{2} \frac{\mathrm{d}}{\mathrm{d} t}\left\langle\boldsymbol{x}^{\prime}(t) \boldsymbol{x}^{\prime}(t)\right\rangle,
$$




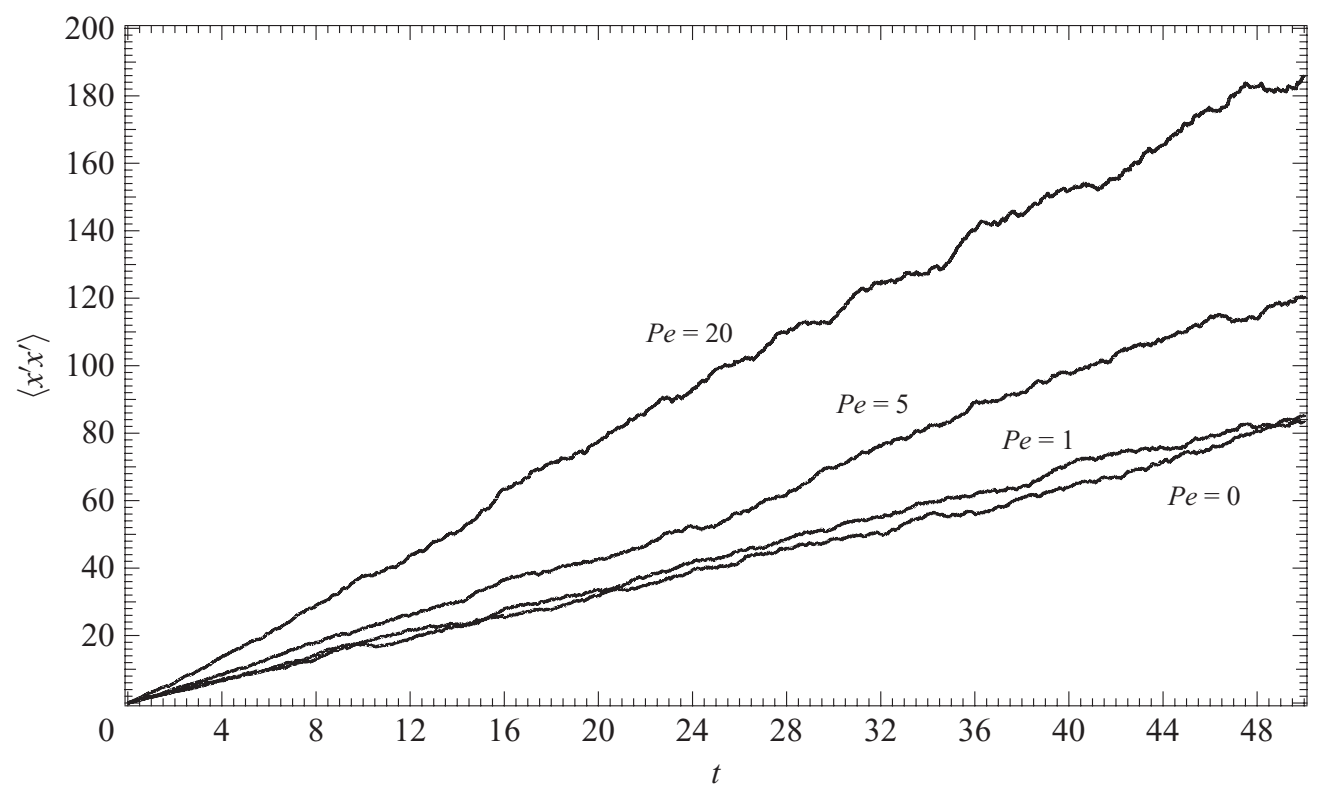

FIGURE 7. Longitudinal mean-square displacement of the probe as a function of time via Brownian dynamics simulation. Volume fraction of bath particles shown is $\phi_{b}=0.1$. Displacements are made dimensionless as $(a+b)$; time is scaled with the correlation time $\tau$, where $\tau \sim a^{2} / D_{a}$ for $P e \leqslant 1$ and $\tau \sim a / U$ for $P e>1$. Each curve is an ensemble average over 480 simulations.

where $\boldsymbol{x}^{\prime} \equiv \boldsymbol{x}(t)-\langle\boldsymbol{x}(t)\rangle$ and the angle brackets \langle\rangle denote an ensemble average over time and over all simulations. A plot of the probe's average mean-square displacement versus time is shown in figure 7, where it can be seen that at long times $\left\langle\boldsymbol{x}^{\prime} \boldsymbol{x}^{\prime}\right\rangle$ grows linearly in time - confirming that the force-induced dispersion of the probe is indeed diffusive, with a constant diffusivity.

To determine the effect of external forcing on the probe's diffusivity, the effective diffusivity in the absence of flow, i.e. at $P e \equiv 0$, is subtracted from $\boldsymbol{D}^{\text {eff }}$ to yield the force-induced diffusion $\boldsymbol{D}^{\text {micro }}$ :

$$
\boldsymbol{D}^{\text {micro }}=\boldsymbol{D}^{\text {eff }}-\boldsymbol{D}^{\text {eff }}(P e=0),
$$

which corresponds to (2.14) defined in text. Results are plotted in figure 8, where $\boldsymbol{D}^{\text {micro }}$ is made dimensionless with probe self-diffusivity $D_{a}$ and scaled with the volume fraction of bath particles $\phi_{b}$.

For $\phi_{b}=0.1$, the Brownian dynamics data match the theoretical solution over the full range of $P e$, but the data for other values of $\phi_{b}$ do not collapse together as expected. The data follow the same qualitative trend, but for $\phi_{b}>0.1$ lie beneath the numerical solution, indicating a dependence on volume fraction - even though the bath is modelled as an ideal gas. This surprising result can be understood by noting that the bath particles' motion is correlated via their interactions with the probe. Although the bath particles do not interact directly, a probe-bath particle collision changes the position of the probe relative to the other nearby particles, giving rise to a correlation between the bath particles. That this correlation must exist can be seen from the dilute pairwise expression for the equilibrium long-time self-diffusivity of a probe equal in size to the bath particles, $D_{\infty}^{s}=\left(1-2 \phi_{b}\right) D_{a}$. This result predicts a negative diffusion coefficient for $\phi_{b}>0.5$ if only pairwise interaction 


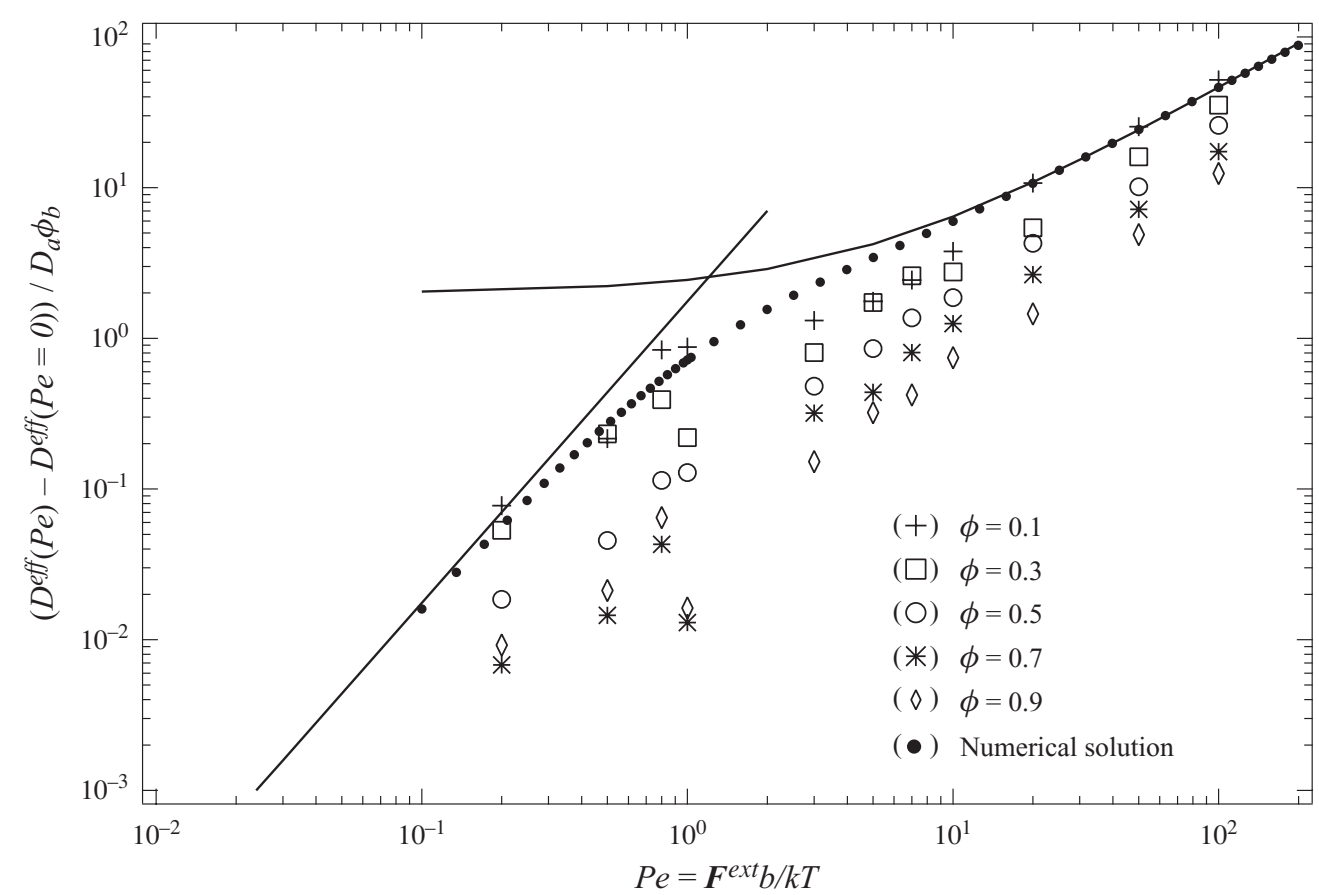

FiguRE 8. Microdiffusivity from Brownian dynamics simulation with ideal gas bath, $a / b=1$, no hydrodynamic interactions, and $\phi_{b}$ as shown in legend, plotted with analytical and numerical solutions of the Smoluchowski equation (only longitudinal direction only is shown). Solution of Smoluchowski equation is shown by solid asymptotes (analytical results) and filled circles (numerical results). Error bars are of the order of marker size; each marker represents a set of 480 simulations.

were important. Clearly the correlated behaviour via interaction with the probe is critical even though the bath particles are an ideal gas. In figure 9 we show the actual long-time self-diffusivity of the probe as determined by Brownian dynamics simulation. Also shown in the figure is a theoretical prediction for $D_{\infty}^{s}$, which follows from the approach of Brady (1994), who determined the long-time self-diffusivity of hard spheres in concentrated suspensions as

$$
D_{\infty}^{s}\left(\phi_{b}\right)=D_{a}\left[1+2 \phi_{b} g_{0}\left(2 ; \phi_{b}\right)\right]^{-1} .
$$

For ideal-gas bath particles the equilibrium pair-distribution function at contact is $g_{0}=1$, and we have

$$
D_{\infty}^{s}\left(\phi_{b}\right)=D_{a} \frac{1}{\left(1+2 \phi_{b}\right)},
$$

which is in reasonable agreement with the results from Brownian dynamics simulations in figure 9 (where $a / b \sim O(1)$ ).

The correlated collisions make the bath more resistive to the motion of the probe as evidenced by the smaller long-time self-diffusivity - in the linear-response regime the diffusivity and the resistance to external forcing are the same - and so what is important is the actual probe speed $\langle U\rangle$ rather than the imposed external force in setting the Péclet number. The probe perturbs the microstructure with this average speed. Away from the probe, the bath particles again form an ideal gas and restore the microstructure under their own Brownian motion which is unhindered. This gives 


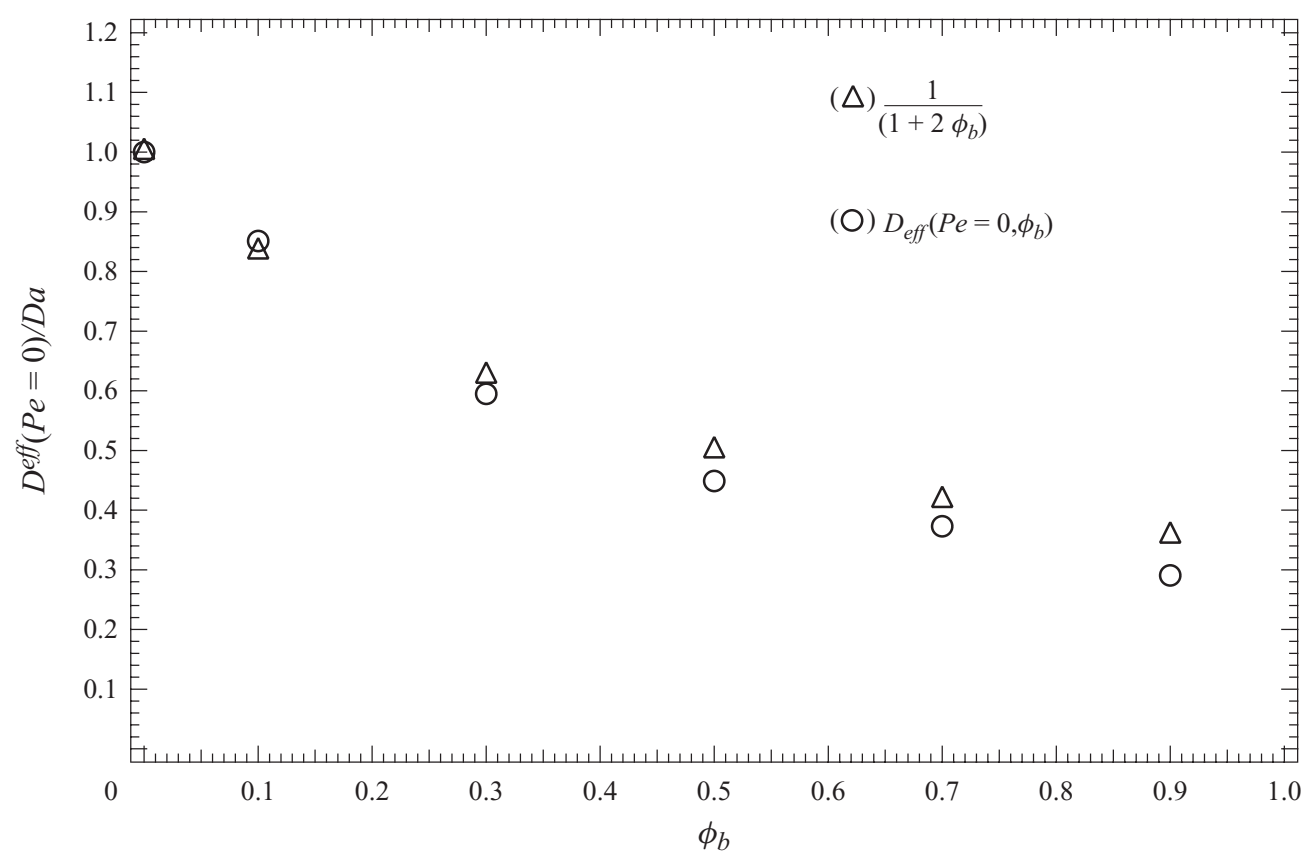

FIGURE 9. Effective diffusivity of the unforced probe for various concentrations of bath particles (probe to bath particle size ratio taken to be unity). (O) Results measured via Brownian dynamics simulation; $(\triangle)$ Results that correspond to theory, $D_{\infty}^{s}=1 /\left(1+2 \phi_{b}\right)$. Error bars are of the order of marker size; each marker represents a set of 480 simulations.

an effective Péclet number:

$$
P e^{e f f}=\frac{\langle U\rangle a}{D_{b}},
$$

where $D_{b}$ is the bare diffusivity of the bath particles away from the probe and $\langle U\rangle$ is the average probe velocity determined by simulation.

The scaling of $D^{\text {micro }}$ with the isolated probe diffusivity $D_{a}$ (the vertical axis in figure 8) emerged from the kinematic expression for probe flux (see (2.3)) where the diffusive flux of the probe was assumed to depend on unhindered probe diffusion. But for small $P e$ the probe forcing should be proportional to $D_{\infty}^{s}$ rather than $D_{a}$ as the deformation to the microstructure is sensitive to the correlated bath particle behaviour. At very large $P e$ (when the boundary layer is thin) the unhindered forcing, $D_{a}$, is appropriate. We apply this rescaling of the Péclet number over the full range of $P e$, and of $D^{\text {micro }}$ for $0 \leqslant P e \lesssim 20$, as seen in figure 10 . The Brownian dynamics results now collapse onto the theoretical results in a universal curve for $a / b=1$.

\section{Comparison to macrodiffusivity}

Previous studies of particle motion in macrorheology show that imposing a shear flow on a colloidal dispersion increases particle diffusivity (Leighton \& Acrivos 1987; Morris \& Brady 1996; Brady \& Morris 1997; Breedveld et al. 1998). In the noncolloidal regime, the dispersivity of a sphere falling through a neutrally buoyant suspension at low Reynolds number and high Péclet number has also been studied both analytically (Davis \& Hill 1992) and experimentally (Abbot et al. 1997). It is 


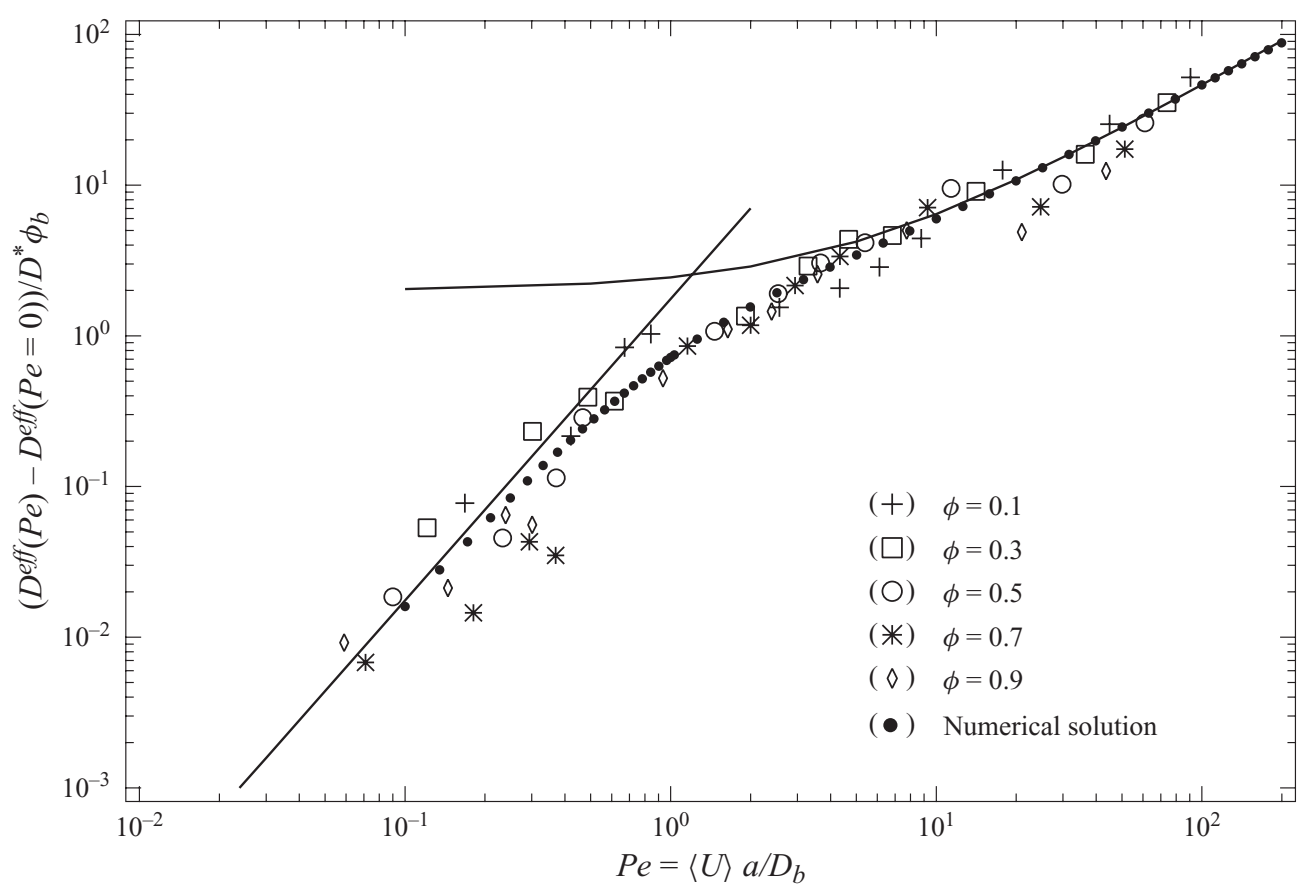

FiguRE 10. Brownian dynamics simulation data, shown with analytical (solid lines) and numerical solution (filled circles) of the Smoluchowski equation (longitudinal). The force-induced microdiffusivity, $D^{\text {micro }}$, is scaled with volume fraction of bath particles, $\phi_{b}$, and made dimensionless with $D^{*}$, which corresponds to $D_{\infty}^{s}$ for $0 \leqslant P e \leqslant 20$ and to $D_{a}$ for $P e>20$. It is plotted against the effective Péclet number, which reflects the correlation of the bath particles. Error bars are of the order of marker size; each marker represents a set of 480 simulations.

of interest to seek comparison between such macroscale behaviour and the results of this investigation.

Both shear-induced macrodiffusivity and force-induced microdiffusivity result from the scattering of a tracer, or probe, particle by the deformed microstructure. Brady \& Morris $(1996,1997)$ determined the shear-induced long-time diffusion tensor $\boldsymbol{D}^{\text {macro }}$ for weak shearing and strong shearing. Both $\boldsymbol{D}^{\text {micro }}$ and $\boldsymbol{D}^{\text {macro }}$ scale as $\sim O\left(\phi_{b} P e\right)$ for $P e \gg 1$. At small values of the Péclet number, the dependence of force-induced diffusion on $P e$ changes in both micro and macro cases as the correlation time scale becomes diffusive; while the former scales as $\sim O\left(\phi_{b} P e^{2}\right)$, the latter scales as $\sim O\left(\phi_{b} P e^{3 / 2}\right)$ - reflecting the different symmetry and forcing of the two microstructures. Further, in both flows the distorted bath microstructure is asymmetric (cf. figure 1), which gives rise to an anisotropic diffusion tensor. Brady \& Morris (1997) also showed that the shear-induced diffusion tensor could be directly related to the bulk stress tensor for the suspension, and its anisotropy to the normal stress differences - a hallmark of the rheological behaviour of far-from-equilibrium complex fluids. We have shown that the microdiffusivity tensor is also anisotropic; the notion of normal stress differences in the context of active microrheology is an intriguing one, and this connection should be explored further.

The settling of a non-Brownian ball through a neutrally buoyant quiescent suspension of non-colloidal spheres is the macroscale analogue of our model at 
very high $P e$ (in the falling-ball regime, the Péclet number is very large due to particle size). The dispersion of the ball's trajectory arises due to many uncorrelated hydrodynamic interactions with the background suspended balls. Davis \& Hill (1991) theoretically determined the hydrodynamic diffusivity of a falling ball in the dilute limit for all size ratios $a / b$. For size ratio of order unity, they give the hydrodynamic diffusivity as

$$
\frac{D_{\text {theory }}^{H}}{U a} \approx 1.33\left(\frac{b}{a}\right)^{2} \phi_{b} .
$$

Here, $U=F^{\text {grav }} / 6 \pi \eta a$ is the Stokes velocity of the falling ball, $F^{\text {grav }}$ is the net force due to gravity, $\eta$ is the solvent viscosity and the superscript $H$ denotes the hydrodynamic diffusivity. Abbot et al. (1997) conducted experiments in which they measured the dispersion of a ball of size $a$ as it fell through a suspension of balls of size $b$, for several volume fractions $\phi_{b}$ and a range of probe-to-background ball size ratios, $a / b$. At the smallest volume fraction measured, $\phi_{b}=0.15$, for $O(1) \lesssim a / b \lesssim O(10)$ they found the diffusivity of the falling ball to be

$$
\frac{D_{\text {meas }}^{H}}{\langle U\rangle a}=1.067\left(\frac{b}{a}\right)^{1.93_{-0.26}^{+0.53}} \phi_{b},
$$

where the error shown corresponds to $95 \%$ confidence limits and $\langle U\rangle$ is the average vertical speed of the falling ball. We recall the $O(P e)$ result for the parallel microdiffusvity from $\S 4.2$ :

$$
\frac{D_{\text {theory }}^{\text {micro }}}{\langle U\rangle a}=\frac{1}{4}\left(\ln 2-\frac{1}{4}\right)\left(\frac{b}{a}\right)\left(1+\frac{a}{b}\right)^{2} \phi_{b},
$$

The hydrodynamic interactions, particularly the near-field lubrication interactions, between the non-colloidal spheres produce a dependence on size ratio that is qualitatively different than the size ratio dependence in the case of no hydrodynamic interactions considered in this study. Hydrodynamic interactions can be included in the theory developed here and is left for a future study. However, the same scaling in $P e_{b}$ and $\phi_{b}$ is obtained.

\section{Summary and concluding remarks}

We have extended the model of active nonlinear microrheology to account for fluctuations in probe motion. A dilute colloidal dispersion of hard spheres through which a Brownian probe is driven by a constant external force was studied. Collisions between the probe and bath particles were shown to drive a long-time diffusive spread of the probe's trajectory - a force-induced diffusion, or 'microdiffusivity'. The microdiffusivity increases the probe's long-time self-diffusion. In the limit $P e \rightarrow 0$, $D^{\text {micro }}$ scales quadratically: $D^{\text {micro }} \sim P e^{2} \phi_{b} D_{a}$. At the opposite extreme where $P e \rightarrow \infty$ the microdiffusivity is linear in the forcing, $D^{\text {micro }} \sim P e \phi_{b} D_{a} \sim\left(F^{e x t} / \eta\right) \phi_{b}$, and the force-induced diffusion dominates the spread of the probe's trajectory.

For all values of $P e$, it was found that the dependence of the microdiffusivity on the volume fraction of bath particles, $\phi_{b}$, is not strictly linear even when only pairwise interactions are considered and the bath particles are an ideal gas. The motion of the bath particles becomes correlated via their interactions with the probe, and when $\phi_{b} \gtrsim 0.1$, the effect of the correlation becomes important. A key effect of the bath particle correlation is a reduction in the mean speed of the probe; since now $\langle U\rangle$ is 
the speed with which the microstructure is perturbed, the appropriate Péclet number is given by $P e^{e f f}=\langle U\rangle a / D_{b}$. Similarly, the diffusive flux of the probe is hindered by the bath particles at small $P e$, but for larger $P e$ its relaxation through the boundary layer is set by its bare diffusion coefficient $D_{a}$. The microdiffusivity should then be made dimensionless by $D_{\infty}^{s}$ for $0 \leqslant P e \lesssim 20$ and by its bare diffusivity $D_{a}$ for $P e \gtrsim 20$, where it is proposed that $D_{\infty}^{s}=D_{a} /\left(1+2 \phi_{b}\right)$ accounts for the correlation. A rescaling of all $P e$ for $\phi_{b}>0.1$ that reflects this physical process collapses the data onto a single curve given by the dilute theory, as shown in figure 10. This correlated behaviour of the bath particles raises the issue of excluded volume interactions between the bath particles, a subject that merits further study.

It was found that for all $P e$, neglecting hydrodynamic interactions, the microdiffusivity is proportional to the scale factor $(1+a / b)^{2}$. In the limit of a small probe, $a / b \rightarrow 0$, this factor reduces to unity and the microdiffusivity scales with $P e$ and $\phi_{b}$ as expected. However, when $a / b$ is large, the analysis predicts a microdiffusivity that grows as $(a / b)^{2}$. This result is counter to what one would expect, because very small bath particles should produce correspondingly small effects. There are two sources for this behaviour. The first is the neglect of hydrodynamic interactions between the probe and the bath particles. A large probe would appear as a rigid surface to the small bath particles and it may be a poor approximation to have neglected the long-range hydrodynamic interactions. The other contributor to the apparent paradox is the way we have stated the standard (macro)rheological condition for diluteness: $\phi_{b} \ll 1$ dictates only that the typical distance between bath particles must be small. But in microrheology, for the probe to move through a dilute bath, it is required that the probe encounter only one bath particle at a time. The probe's contact area scales as $a^{2}$, and the number of colliders $n_{b}$ within one collision distance, $b$, must be small, that is, a shell of thickness $b$ around the probe can contain at most one bath particle at any given time in order to assure that only pair collisions occur: $n_{b} a^{2} b \ll 1$. Hence, the diluteness condition is more properly stated as $\phi_{b}(a / b)^{2} \ll 1$. With this constraint, the microdiffusivity, e.g. at large $P e$, is $D^{\text {micro }} \sim(a / b)^{2} \phi_{b} D_{a} P e$ and remains small for $a / b \gg 1$.

Next, a comparison between tracer motion in a sheared suspension and probe motion in active microrheology showed that both flows give rise to an enhancement to long-time self-diffusivity. Further, for both systems the asymmetry of the deformed microstructure produces an anisotropic diffusion tensor, which prompts the question of normal stress differences. Understanding the close qualitative relationship between macrodiffusivity and microdiffusivity and their connection to normal stress differences can enable the use of the latter as a material interrogation technique - e.g. the measurement of the long-time self-diffusion of the probe may yield normal stresses, a subject of a future study.

The comparison between falling-ball rheometry in the dilute limit and active microrheology in the dual limit $P e \rightarrow \infty, \phi_{b} \ll 1$ shows that in both regimes the diffusivity of the probe scales linearly in both the forcing and the volume fraction of bath particles. A key physical difference between the two problems is that the fallingball dispersivity is a purely hydrodynamic phenomenon, whereas our model for active microrheology excludes all but hard-sphere mechanical interactions. This difference manifests in the dependence of force-induced diffusion on probe/background-particle size ratio $a / b$. The effect of hydrodynamic interactions between colloidal particles is not expected to change the scaling in $\phi_{b}$, but is likely to yield a different dependence on particle size ratio.

Indeed, an important area for future study is how hydrodynamic interactions affect the force-induced diffusion. When hydrodynamic interactions are important, 
the probe flux changes; its ensemble average (see (2.7)) now contains an additional term, $\boldsymbol{R}_{F U}^{-1} \cdot \boldsymbol{F}^{P} \hat{P}_{1}$. As before, the terms in the average probe flux can be arranged to more readily identify the physical process associated with each one (cf. (2.11)). The new hydrodynamic contribution appears in the first bracketed term, which is a coefficient of the probability density $\hat{P}_{1}$ - hydrodynamics affect the probe's average speed. Furthermore, since the bare diffusion tensor for the probe, $\boldsymbol{D}_{a}$, now depends on relative positions of probe and bath particles, it remains inside the integrals in (2.11). This gives only a geometric scale-factor difference in the general expression for the effective diffusivity, (2.12). Now for large $P e$, hydrodynamic interactions also qualitatively alter the deformed microstructure; the scaling of $g_{0}$ in $P e$ varies in the presence of hydrodynamic interactions. In the so-called pure hydrodynamic limit, $a / a_{h} \equiv 1$ (Khair \& Brady 2006), the scaling becomes $g_{0} \sim P e^{0.78}$, and varies continuously up to $g_{0} \sim P e$ for $a / a_{h} \rightarrow \infty$; a similar qualitative change in $P e$-scaling is expected for the deflection field. Thus the presence of hydrodynamic interactions will give rise to a quantitative change in the bare diffusion coefficient and at high $P e$, a qualitative change in the deflection field $\boldsymbol{d}$ (cf. (3.9)-(3.11)). Since the microdiffusivity is the first moment of the deflection field at contact, its scaling in $P e$ is expected to change accordingly. And as noted above, significant qualitative changes in the size-ratio dependence (not in $P e$ ) can occur at high $P e$ in the case of disparate probe-to-bath-particle size ratio.

Often the particles studied experimentally have a shape that deviates from that of a sphere - sometimes significantly so. An ellipsoidal-type probe particle in a dispersion of spherical bath particles can be studied, where an external force is applied through the probe's centre. The new length scales added by the minor and major axes of the probe gives rise to a quantitative difference in the diffusivity - the size dependence of the diffusivity would reflect the new length scales. One would also need to consider the importance of rotational diffusion. As the probe moves through the bath, the angle its major axis forms with the line of action of the external force changes. For small $\mathrm{Pe}$, all orientations are equally likely; the rotational diffusion would make the probe act as a 'sphere' of size $R_{\text {avg }}$, the rotational average of the major and minor axes, and thus still give the same forcing (dipolar microstructural disturbance). The scaling in $P e$ is identical, since the flow is the same. At high $P e$, rotational diffusion is slow compared to advection, and one expects the probe particle to attain a stable orientation with its major axis transverse to the forcing, as found by Khair \& Brady (2008) for a constant-velocity ellipsoidal-type probe. They also found that the perturbation to the microstructure scales linearly in $P e$ for $P e \gg 1$ in the absence of hydrodynamics, and so the scaling in $P e$ remains the same. For intermediate values of $P e$, in the case of constant forcing, rotational diffusion matters. But one would predict that a balance in kicks from bath particles would again align the probe transverse to the flow as at high $\mathrm{Pe}$. Thus, the contribution to the diffusion coefficient is at most a geometric scaling of the size dependence.

An important outcome of this study is its general applicability to a wider class of problems. Thus far we have studied the diffusive motion of a particle moving due to an externally applied force through a bath of other particles. Many systems of interest, however, consist of self-propelled objects; biological microswimmers are important examples. Can such motion be related to that of the externally forced particle studied here? In fact, it turns out that the results obtained here are the same as those one would obtain for many self-propelled particles. What is important is the relative motion of the probe to the bath particles; so if one can neglect hydrodynamic interactions, the results in the case of a microscale swimmer would 
be identical to those obtained in this study (with $P e$ based on $U_{\text {probe }}$ rather than an external force). A self-propelled object must be torque- and force-free; so if the object is a swimmer, velocity decays as $\sim 1 / r^{2}$. Thus, neglecting hydrodynamic interactions may be a reasonable approximation. In the case of electrophoretic motion, the velocity disturbance decays as $\sim 1 / r^{3}$ and neglecting hydrodynamics is an even better approximation. (Including hydrodynamics would make only a quantitative difference at small $P e$, as discussed above.) The solution presented for the microdiffusivity is therefore applicable to a very wide range of problems in which hydrodynamic interactions can be neglected - both externally forced and self-propulsive systems. Important future work includes experimental verification of this idea, development of theory for general-shape swimmers and extension to groups of swimmers or propelled objects. The crowded interior of a cell also provides a fascinating opportunity to combine the effects of a concentrated bath with self-propulsion to study the motion of objects diffusing through the intracellular fluid.

Several other interesting questions remain. We have investigated the steady-state behaviour of the suspension, but its transient behaviour is as yet unexplored. Other open questions include the connection of diffusivity to normal stress differences, and the 'continuum' limit for large size ratios $a / b \gg 1$. Notwithstanding, the simplifications invoked in the model thus far provide important conclusions about the fluctuating motion of a tracer particle in the active nonlinear microrheology regime. That this motion is diffusive, with a simple dependence on few parameters, and widely applicable to a range of problems may open new techniques for researchers in material science, the biosciences and nanoscale technology.

This work was supported in part by the National Defense Science and Engineering Graduate Fellowship program and by a grant from the National Science Foundation. The authors gratefully acknowldege James W. Swan for Brownian dynamics simulations code for nonlinear microrheology, and Manuj Swaroop for assistance in the finite difference solutions of the Smoluchowski equation.

\section{REFERENCES}

Aвbot, J. R., Graham, A. L., Mondy, L. A. \& Brenner, H. 1997 Dispersion of a ball settling through a quiescent neutrally buoyant suspension. J. Fluid Mech. 361, 309-331.

BAtchelor, G. K. 1976 Brownian diffusion of particles with hydrodynamic interaction. J. Fluid Mech. 74, 1-29.

Bergenholtz, J., Brady, J. F. \& Vicic, M. 2002 The non-Newtonian rheology of dilute colloidal dispersions. J. Fluid Mech. 456, 239-275.

Brady, J. F. 1994 The long-time self-diffusivity in concentrated colloidal dispersions. J. Fluid Mech. 272, 109-133.

Brady, J. F. \& Morris, J. F. 1997 Microstructure of strongly sheared suspensions and its impact on rheology and diffusion. J. Fluid Mech. 348, 103-139.

Breedveld, J., van den Ende, D., Tripathi, A. \& Acrivos, A. 1998 The measurement of the shear-induced particle and fluid tracer diffusivities in concentrated suspensions by a novel method. J. Fluid Mech. 375, 297-318.

Carpen, I. C. \& Brady, J. F. 2005 Microrheology of colloidal dispersions by Brownian dynamics simulations. J. Rheol. 49, 1483-1502.

Davis, R. H. \& HiLl, N. A. 1992 Hydrodynamic diffusion of a sphere sedimenting through a dilute suspension of neutrally buoyant spheres. J. Fluid Mech. 236, 513-533.

Habdas, P., Schaar, D., Levitt, A. C. \& Weeks, E. R. 2004 Forced motion of a probe particle near the colloidal glass transition. Europhys. Lett. 67, 477-483.

Heath, J. R., Davis, M. E. \& Hood, L. 2009 Nanomedicine: revolutionizing the fight against cancer. Sci. Am. 300, 44. 
Heyes, D. M. \& Melrose, J. R. 1993 Brownian dynamics simulations of model hard-sphere suspensions. J. Non-Newton. Fluid Mech. 46, 1-28.

Janke, C., Rogowski, K., Wloga, D., Regnard, C., Kajava, A. V., Strub, J. M., Temurak, N., van Dijk, J., Boucher, D., van Dorseelaer, A., Suryavanshi, S., Gaertig, J. \& Edde, B. 2005 Tubulin polyglutamylase enzymes are members of the TTL domain protein family. Science 308, 1758-1762.

Khair, A. S. \& Brady, J. F. 2006 Single particle motion in colloidal dispersions: a simple model for active and nonlinear microrheology. J. Fluid Mech. 557, 73-117.

Khair, A. S. \& Brady, J. F. 2008 Microrheology of colloidal dispersions: shape matters. J. Rheol. 52, 165-196.

Leighton, D. \& ACRivos, A. 1987 Measurement of shear-induced self-diffusion in concentrated suspensions of spheres. J. Fluid Mech. 177, 109-131.

MacKintosh, F. C. \& Schmidt, C. F. 1999 Microrheology. Curr. Opin. Colloid Interface Sci. 4, 300-307.

Meyer, A., Marshall, A., Bush, B. G. \& Furst, E. M. 2005 Laser tweezer microrheology of a colloidal suspension. J. Rheol. 50, 77-92.

Morris, J. F. \& Brady, J. F. 1996 Self-diffusion in sheared suspensions. J. Fluid Mech. 312, 223-252.

Shirai, Y., Osgood, A. J., Zhao, Y., Kelly, K. F. \& Tour, J. M. 2005 Directional control in thermally driven single-molecule nanocars. Nano Lett. 5, 2330-2334.

SQUiRES, T. M. 2008 Nonlinear microrheology: bulk stresses versus direct interactions. Langmuir 24, $1147-1159$.

Squires, T. M. \& Brady, J. F. 2005 A simple paradigm for active and nonlinear microrheology. Phys. Fluids 17, 073101-1-073101-2.

Wilson, L. G., Harrison, A. W., Schofield, A. B., Arlt, J. \& Poon, W. C. K. 2009 Passive and active microrheology of hard-sphere colloids. J. Phys. Chem. 113, 3806-3812. 